A. Latz, J. Zausch

Thermodynamic consistent transport theory of Li-lon batteries 
(C) Fraunhofer-Institut für Techno- und Wirtschaftsmathematik ITWM 2010

ISSN 1434-9973

Bericht 195 (2010)

Alle Rechte vorbehalten. Ohne ausdrückliche schriftliche Genehmigung des Herausgebers ist es nicht gestattet, das Buch oder Teile daraus in irgendeiner Form durch Fotokopie, Mikrofilm oder andere Verfahren zu reproduzieren oder in eine für Maschinen, insbesondere Datenverarbeitungsanlagen, verwendbare Sprache zu übertragen. Dasselbe gilt für das Recht der öffentlichen Wiedergabe.

Warennamen werden ohne Gewährleistung der freien Verwendbarkeit benutzt.

Die Veröffentlichungen in der Berichtsreihe des Fraunhofer ITWM können bezogen werden über:

Fraunhofer-Institut für Techno- und Wirtschaftsmathematik ITWM Fraunhofer-Platz 1

67663 Kaiserslautern

Germany

Telefon: $\quad+49(0) 631 / 31600-0$

Telefax: $\quad+49(0) 631 / 31600-1099$

E-Mail: info@itwm.fraunhofer.de

Internet: www.itwm.fraunhofer.de 
Das Tätigkeitsfeld des Fraunhofer-Instituts für Techno- und Wirtschaftsmathematik ITWM umfasst anwendungsnahe Grundlagenforschung, angewandte Forschung sowie Beratung und kundenspezifische Lösungen auf allen Gebieten, die für Techno- und Wirtschaftsmathematik bedeutsam sind.

In der Reihe »Berichte des Fraunhofer ITWM « soll die Arbeit des Instituts kontinuierlich einer interessierten Öffentlichkeit in Industrie, Wirtschaft und Wissenschaft vorgestellt werden. Durch die enge Verzahnung mit dem Fachbereich Mathematik der Universität Kaiserslautern sowie durch zahlreiche Kooperationen mit internationalen Institutionen und Hochschulen in den Bereichen Ausbildung und Forschung ist ein großes Potenzial für Forschungsberichte vorhanden. In die Berichtreihe werden sowohl hervorragende Diplom- und Projektarbeiten und Dissertationen als auch Forschungsberichte der Institutsmitarbeiter und Institutsgäste zu aktuellen Fragen der Techno- und Wirtschaftsmathematik aufgenommen.

Darüber hinaus bietet die Reihe ein Forum für die Berichterstattung über die zahlreichen Kooperationsprojekte des Instituts mit Partnern aus Industrie und Wirtschaft.

Berichterstattung heißt hier Dokumentation des Transfers aktueller Ergebnisse aus mathematischer Forschungs- und Entwicklungsarbeit in industrielle Anwendungen und Softwareprodukte - und umgekehrt, denn Probleme der Praxis generieren neue interessante mathematische Fragestellungen.

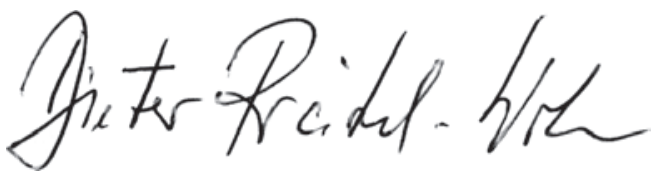

Prof. Dr. Dieter Prätzel-Wolters Institutsleiter

Kaiserslautern, im Juni 2001 



\title{
Thermodynamic consistent transport theory of Li-Ion batteries
}

\author{
A. Latz, J. Zausch \\ Fraunhofer Institut für Techno- und Wirtschaftsmathematik \\ Kaiserslautern, Germany
}

August 25, 2010

\begin{abstract}
We will present a rigorous derivation of the equations and interface conditions for ion, charge and heat transport in Li-ion insertion batteries. The derivation is based exclusively on universally accepted principles of nonequilibrium thermodynamics and the assumption of a one step intercalation reaction at the interface of electrolyte and active particles. Without loss of generality the transport in the active particle is assumed to be isotropic. The electrolyte is described as a fully dissociated salt in a neutral solvent. The presented theory is valid for transport on a spatial scale for which local charge neutrality holds i.e. beyond the scale of the diffuse double layer. Charge neutrality is explicitely used to determine the correct set of thermodynamically independent variables. The theory guarantees strictly positive entropy production. The various contributions to the Peltier coefficients for the interface between the active particles and the electrolyte as well as the contributions to the heat of mixing are obtained as a result of the theory.
\end{abstract}

keywords: Li Ion Batteries,Nonequilibrium Thermodynamics,Thermal Transport,Modeling

\section{Introduction}

Understanding the heat generation and temperature distribution in Li ion battery cells is of great practical interest. Many degradation processes in Li ion batteries are caused by and most of them are enhanced under temperature increase $[1,2]$. Thermal runaway may begin as a very local phenomenon by initiating an exothermal reaction at a local hotspot. 
Therefore the average temperature of a cell is not sufficient to determine the begin of a thermal runaway. A spatially resolved mathematical description of the processes on the scale of the cell would allow to simulate the behavior of the cell under varying operating conditions and to detect the probability of dangerous hotspots.

Mathematical modeling of Li-ion batteries on cell resolved level was pioneered by the work of Newman and his coworkers $[3,4,5]$ and extendend and refined by many other authors $[6,7,8]$. The modeling approaches are based on transport equations for Li ions and charges in the electrolyte as well as in the active particles of cathode and anode under isothermal conditions. Most modeling approaches for the thermal behavior of batteries are concentrating on overall thermal balance equations for a whole cell $[9,10,11]$ $[12,13,14]$ by combining phenomenologically thermodynamic considerations on entropy or enthalpy changes within a cell with reasonable assumptions on out of equilibrium processes like Joule heating, heat of mixing, Peltier effect and Soret effect. An approach based on local continuity equations for the temperature was presented in [15], extending previous work on species and charge transport in batteries [16]. These authors focused on deriving macroscopic equations for the heat transport in porous electrodes using the volume averaging technique. The microscopic equation for the temperature was formulated without considering possible changes of the transport equations describing species and charge transport. Also the interface conditions have not been derived. Fully coupled models have been considered for battery stacks [17] and other types of batteries (see e.g. $[18,19])$. Also in these cases the equations were not derived but formulated as balance equations in which known physical effects as well as the reaction kinetics for all relevant chemical reactions were phenomenologically incorporated.

A different approach to treat transport in electrochemical systems is the systematic approach of nonequilibrium thermodynamics [20] pioneered by Landau [21, 22]. In this approach only conservation equations and general principles of statistical mechanics were used to derive the general form of the relevant transport equations. Only measurable quantities like thermodynamic derivatives and transport coefficients enter the equations. The Onsager relations which guarantee the positivity of the entropy production and impose strict relations between the equations for densities, charges and temperature, play a central part in the derivation. Chemical reactions can easily be treated within this formalism [20]. The interface conditions can also be derived by necessary continuity constraints (as e.g conservation of charges, energy etc. ) and considering the physical and chemical processes within the interface [20]. Contrary to the phenomenological approaches the terms contributing to the heat production are systematically derived using nothing else but generally valid thermodynamic relations and total energy conservation, instead of just physical intuition and experimental experience. In this manner it is made certain that no source of heat is neglected, if all hydrodynamic variables and all relevant chemical 
reactions are taken into account.

An especially elegant form of this formalism was recently used to derive a hydrodynamic theory of electromagnetic field in continuous media [23, 24, 25, 26], which can also be applied to charged and magnetic liquids [27]. Also charge and species transport in Li ion batteries can be dealt with using the nonequilibrium thermodynamics framework [28]. We will derive in the following the complete set of equations including all interface conditions for the ion, charge and thermal transport in Li Ion battery cells. We will consider a spatial scale $L$ which is large compared to the scale $L_{D L}$ of the diffuse double layer (i.e $L \gg L_{D L} \sim 10-20 \mathrm{~nm}$ [4] ). Concentrated electrolytes are neutral on this scale due to the strong Coulomb interactions between the ions leading to an extremely small Debye screening length. Potential gradients are only in the double layer around the active particles strong enough to cause charge separation [4]. Also active particles fulfill charge neutrality although the mechanism for obtaining charge neutrality is very different. In active particles it is the large mobility of electrons which guarantees charge neutrality. The charge of an inserted Li Ions is instantaneously shielded by local rearrangements of electronic charges and the transport of electrons into the active particles over the current collectors. In the electrolyte charge neutrality will lead to highly correlated motion of negative and positive ions. We will make explicit use of charge neutrality in the derivation of our equations.

In addition to naturally reducing the numbers of relevant equations, the use of charge neutrality also guarantees, that the transport coefficients appearing in theory are the measurable quantities of the electrolyte. Let us for example consider the diffusion processes in a molten salt consisting of positive and negative ions. In general the self diffusion coefficients are different for the two kinds of ions. If there were no interaction between the ions, we could expect to obtain free electric charges in relaxation processes even if the initial perturbation were neutral due to the different diffusion length of the ions. In reality the strong Coulomb interaction between the ions prevents the appearance of free charges. The process relevant for diffusion is in fact the collective interdiffusion process with a uniquely defined interdiffusion coefficient for both species. This unique quantity for positive and negative ions will appear naturally in our theory.

To derive the transport equations in the electrolyte and the solid particles we use a general and rigorous theory for polarizable, conducting media introduced in [23] and generalized in [24]. The theory is based on general thermodynamic principles, Maxwells theory for electromagnetic fields and Onsagers reciprocal relations. Although transport mechanisms in active particles and electrolyte can be very different on the microscopic (atomic) scale, their macroscopic form just differ in the functional dependencies and the size of the transport coefficients. For example in active particles charge is transported mainly by pure electronic conduction. The contribution of the ion diffusion in the active 
particles to the electric current can be neglected due to the large mobility of the electrons compared to the ions. Charge transport in the electrolyte on the other side is exclusively due to ionic transport. In fact the transfer of electrons into the electrolyte would result in the reduction of $\mathrm{Li}$ ions in the electrolyte to metallic Lithium and is considered to be one of the many degradation mechanisms in Li ion batteries [1]. These huge differences in the microscopic charge transport mechanisms appear macroscopically just as different meaning of the respective charge conductivity coefficients. In electrolytes it is interpreted as concentration dependent ionic conductivity in the active particle we may use a constant electronic conductivity. In addition the transfer number of ions in active particles can be set to zero due to the vanishing contribution of ion diffusion to the electrical current.

\section{Model}

Originally the theory of Henjes and Liu [23] was used to derive the hydrodynamic equations for a one component polarizable liquids. But the principles can easily be applied to a mixture of a dissociating salt and a solvent i.e for a generic electrolyte as used in batteries. The reduction to the transport in the solid particle is straight forward after the full set of equations for the electrolyte are derived. Although we have to deal with a at least three component system of positive and negative ions as well as neutral solvent molecules, the problem can be reduced to an effective one component problem using two constraints, which are applicable to the physical situation of a concentrated electrolyte in a battery. The first observation is that convective transport is highly unlikely under normal operating conditions in a battery. We therefore may safely assume that the center of mass of any hydrodynamic volume element is at rest. This assumption allows to eliminate the concentration of the neutral solvent as independent variable. With $M_{0}, M_{+}, M_{-}$ being the molar masses of solvent and positive and negative ions respectively we get in the absence of convection the relations

$$
M_{0} d c_{0}+M_{+} d c_{+}+M_{-} d c_{-}=0
$$

for the changes in the respective concentrations.

The second observation concerns the occurrence of finite free charges in a volume element. It can easily be estimated [4] that the electric fields which where necessary to impose charge separation on a micrometer scale are much bigger than the average fields present in a battery. Charge separation is only observed in the double layer around active particles on a scale of the order of $10-20 \mathrm{~nm}$. The present theory will be restricted to scales above $100 \mathrm{~nm}$. A detailed theory for the linear and nonlinear phenomena in the 
double layer can be found in $[29,30]$. If we denote molar concentrations of positive and negative ions of charge $z_{+}$and $z_{-}$with $c_{+}$and $c_{-}$, respectively, charge neutrality requires

$$
\nu_{+} z_{+} c_{+}+\nu_{-} z_{-} c_{-}=0
$$

where $\nu_{+}, \nu_{-}$are the stoichiometric numbers of positive and negative charges. It is therefore sufficient to determine the transport equations for the concentration $c=c_{+}=$ $-\frac{\nu_{-} z_{-}}{\nu_{+} z_{+}} c_{-}$. For notational simplicity we restrict our theory to a binary salt with $\nu_{+}=$ $\nu_{-}=1$ and consequently $z_{-}=-z_{+}$as well as

$$
c_{+}=c_{-}
$$

The fundamental thermodynamic relation for a polarizable system in an external electromagnetic field is given by [23]

$$
d e=T d s+\mu_{+} d c_{+}+\mu_{-} d c_{-}+\mu_{0} d c_{0}+\vec{E} d \vec{D}+\vec{H} d \vec{B}
$$

Here $e, s, \mu_{i}$ are the energy density, entropy density and the chemical potential of species i. $\vec{E}, \vec{D}, \vec{B}, \vec{H}$ are electric field, electric displacement, magnetic induction and magnetic field respectively. Using (1) and (3), Eq. (4) reduces to

$$
d e=T d s+\mu d c+\vec{E} d \vec{D}+\vec{H} d \vec{B}
$$

where $c \equiv c_{+}$is the concentration of Li - ions and the effective chemical potential $\mu$ of the Lithium ions is given by

$$
\mu=\mu_{+}-\frac{M_{+}}{M_{0}} \mu_{0}-\frac{z_{+}}{z_{-}}\left(\mu_{-}-\frac{M_{-}}{M_{0}} \mu_{0}\right)
$$

For a binary charge neutral salt this reduces to

$$
\mu=\mu_{+}+\mu_{-}-\frac{M_{-}+M_{+}}{M_{0}} \mu_{0}
$$

The next building blocks are the conservation equation for concentration of Li ion, charge density $\rho_{e l}$, entropy as well as the Maxwell equations. Note that the macroscopic velocity is set to zero, due to the main assumption of convection free transport in Li ion batteries. The equation for $\rho_{e l}$ is obtained from the conservation equations of ions:

$$
\frac{\partial c_{+}}{\partial t}=-\vec{\nabla} \vec{N}_{+}
$$




$$
\frac{\partial c_{-}}{\partial t}=-\vec{\nabla} \vec{N}_{-}
$$

Using (3) we obtain with $q=z_{+} c_{+}+z_{-} c_{-}$and the definition of the electric current $\vec{j}=z_{+} \vec{N}_{+}+z_{-} \vec{N}_{-}$

$$
\frac{\partial \rho_{e l}}{\partial t} \equiv 0=-\vec{\nabla} \vec{j}
$$

From the Maxwell equation follows

$$
\vec{E} \partial_{t} \vec{D}+\vec{H} \partial_{t} \vec{B}=-\vec{\nabla}(\vec{E} \times \vec{H})-\vec{j} \vec{E}
$$

The general form of the entropy equation is given by

$$
\partial_{t} s=-\vec{\nabla}\left(\frac{q}{T}\right)+\frac{R}{T}
$$

The entropy production $R$ can be derived by combining the first law (5) and the conservation of the total energy. From the first law (5) we obtain

$$
\partial_{t} e=T \partial_{t} s+\mu \partial_{t} c+\vec{E} \partial_{t} \vec{D}+\vec{H} \partial_{t} \vec{B}
$$

With (8), (11) and (12), Eq. (13) transforms into

$$
\partial_{t} e=-\vec{\nabla}\left(\vec{q}+\vec{N}_{+} \mu+\vec{E} \times \vec{H}\right)+R+\vec{q} \frac{\vec{\nabla} T}{T}-\vec{j} \vec{E}+\vec{N}_{+} \vec{\nabla} \mu
$$

Due to the conservation of total energy the change in the local energy has to fulfill a continuity equation i.e.

$$
\partial_{t} e=-\vec{\nabla} \vec{J}_{e}
$$

By comparing (14) and (15) we obtain the entropy production $\mathrm{R}$ as

$$
R=-\vec{q} \frac{\vec{\nabla} T}{T}-\vec{N}_{+} \vec{\nabla} \mu+\vec{j} \vec{E}
$$

The entropy production has to be strictly positive or zero. This requirement imposes strong constraints on the constitutive relations for the fluxes $\vec{q}, \vec{N}_{+}$and $\vec{j}$. Since the chemical potential is difficult to measure it is more convenient to work with $c, T$ and $\vec{E}$ as independent variable. We therefore rewrite equation (16) as

$$
R=-\vec{Q} \frac{\vec{\nabla} T}{T}-\left(\frac{\partial \mu}{\partial c}\right) \vec{N}_{+} \vec{\nabla} c+\vec{j} \vec{E}
$$


with $\vec{Q}=\vec{q}+\vec{N}_{+} T\left(\frac{\partial \mu}{\partial T}\right)$. The expression for the entropy production defines the independent thermodynamic fluxes $\frac{\vec{Q}}{T},\left(\frac{\partial \mu}{\partial c}\right) \vec{N}_{+}$and $\vec{j}$, for which constitutive equations have to be formulated. In order to guarantee positiveness of the entropy production $\mathrm{R}$, the constitutive equations have to be chosen such, that the expression for $R$ (17) is a positive definite binary form. The most general expression for the fluxes is therefore given by

$$
\left(\begin{array}{c}
-\left(\frac{\partial \mu}{\partial c}\right) \vec{N}_{+} \\
\vec{j} \\
-\frac{\vec{Q}}{T}
\end{array}\right)=\left(\begin{array}{ccc}
\left(\frac{\partial \mu}{\partial c}\right) \alpha & -\left(\frac{\partial \mu}{\partial c}\right) \frac{\kappa t_{+}}{F z_{+}} & \left(\frac{\partial \mu}{\partial c}\right) \nu \\
-\left(\frac{\partial \mu}{\partial c}\right) \frac{\kappa t_{+}}{F z_{+}} & \kappa & -\beta \kappa \\
\left(\frac{\partial \mu}{\partial c}\right) \nu & -\beta \kappa & \frac{\gamma}{T}
\end{array}\right)\left(\begin{array}{c}
\vec{\nabla} c \\
\vec{E} \\
\vec{\nabla} T
\end{array}\right)
$$

The symmetry of the Onsager matrix in Eq. (18) is necessary for the positiveness of the entropy production and a consequence of fundamental principles of nature [20]. The physical meaning of the various transport coefficients $\alpha, \kappa, \gamma, \beta, \nu, t_{+}$is getting more obvious after transforming equivalently the constitutive equations into the more traditional form

$$
\begin{aligned}
\vec{N}_{+} & =-D \vec{\nabla} c+\frac{t_{+}}{z_{+} F} \vec{j}-\frac{D c k_{T}}{T} \vec{\nabla} T \\
\vec{j} & =\kappa \vec{E}-\kappa \frac{t_{+}}{z_{+} F}\left(\frac{\partial \mu}{\partial c}\right) \vec{\nabla} c-\beta \kappa \vec{\nabla} T \\
\vec{Q} & =-\lambda \vec{\nabla} T+\beta T \vec{j}+k_{T} c\left(\frac{\partial \mu}{\partial c}\right)\left(\vec{N}_{+}-\frac{t_{+}}{z_{+} F} \vec{j}\right)
\end{aligned}
$$

In this form the entropy production has the simple form

$$
R=\lambda \frac{\left.(\vec{\nabla} T)^{2}\right)}{T}+\frac{\vec{j}^{2}}{\kappa}+\left(\frac{\partial \mu}{\partial c}\right) \frac{\left(\vec{N}_{+}-\frac{t_{+}}{F z_{+}} \vec{j}\right)^{2}}{D}
$$

In order to be positive each term in the expression (22) has to be positive i.e. the heat conductivity $\lambda$, the electric conductivity $\kappa$ and the interdiffusion coefficient $D$ have to be positive.

The electric conductivity $\kappa$ in the electrolyte is a pure ionic conductivity. In addition to the ionic conductivity, the transference number $t_{+}$of Li ions and the Seebeck coefficient $\beta$ are the relevant transport coefficients for the electric current. The transference number also appears in the relation for the ionic flux $\vec{N}_{+}$and determines the amount of ion flux contributed by the electric current. The Seebeck coefficient has the units Volt/Kelvin and is related to the Peltier coefficient $\Pi$ by $\Pi=T \beta$. It quantifies the thermoelectric effect in the electrolyte or the the active particles. The Seebeck coefficient can in principle be positive or negative. 
The two additional transport coefficients relevant for the ionic flux are the inter diffusion coefficient $D$ and the Soret coefficient $k_{T}$. The interdiffusion coefficient $D$, appearing in the relation for the ionic flux $\vec{N}_{+}$, is measured in the limit of zero electrical current and zero temperature gradient. It is given by

$$
D=\alpha-\left(\frac{\partial \mu}{\partial c}\right) \kappa\left(\frac{t_{+}}{F z_{+}}\right)^{2}
$$

Since $D$ has to be positive the Onsager coefficient $\alpha$ and the transference number fulfill an obvious inequality relation. The Soret coefficient $k_{T}$ quantifies thermodiffusional processes and is given by

$$
\frac{D c k_{T}}{T}=\nu-\kappa \frac{\beta t_{+}}{F z_{+}}
$$

Usually the Soret coefficient is of order $0.6-1.5$ [31], but the overall size of the Soret effect is believed to be very small in liquids and electrolytes [32]. Therefore the ion flux induced by temperature gradients is neglected compared to the one caused by electric currents and gradients in concentrations.

The last transport coefficient is the thermal conductivity $\lambda$ measured in the limit of vanishing electric current and vanishing ionic flux. It is given by

$$
\lambda=\gamma-\left(\frac{\partial \mu}{\partial c}\right) \frac{D}{T}\left(k_{T} c\right)^{2}-\kappa T \beta^{2}
$$

It also has to be strictly positive in order to guarantee positive entropy production $R$.

\subsection{Heat transport}

The equation for the temperature can be derived from the entropy balance (12) and the expression for the entropy production (17) and the relation

$$
T \partial_{t} s=c_{p} \rho \partial_{t} T+\left.T \frac{\partial s}{\partial c}\right|_{T} \partial_{t} c
$$

where $c_{p}$ and $\rho$ are the specific heat per unit mass and the mass density of the electrolyte or the active particles. Using the thermodynamic relation $\left.\frac{\partial s}{\partial c}\right|_{T}=-\left.\frac{\partial \mu}{\partial T}\right|_{c}$ and the continuity equation (8) we obtain

$$
c_{p} \rho \partial_{t} T=-\vec{\nabla} \vec{q}-\vec{N}_{+} \vec{\nabla} \mu+\vec{j} \vec{E}-T\left(\frac{\partial \mu}{\partial T}\right) \vec{\nabla} \vec{N}_{+}
$$


Transforming from the original heat flux $\vec{q}$ to the renormalized heat flux $\vec{Q}$ we get

$c_{p} \rho \partial_{t} T=-\vec{\nabla}\left(\vec{Q}-T \vec{N}_{+}\left(\frac{\partial \mu}{\partial T}\right)\right)-\vec{N}_{+}\left(\frac{\partial \mu}{\partial c}\right) \vec{\nabla} c-\vec{N}_{+}\left(\frac{\partial \mu}{\partial T}\right) \vec{\nabla} T+\vec{j} \vec{E}-T\left(\frac{\partial \mu}{\partial T}\right) \vec{\nabla} \vec{N}_{+}$

The third and last term on the right hand side of Eq. (28) are cancelled by the first one on the right hand side, if we neglect the spatial variation of the thermodynamic derivative $\left(\frac{\partial \mu}{\partial T}\right)$. The temperature equation is therefore given by

$$
c_{p} \rho \partial_{t} T=-\vec{\nabla} \vec{Q}-\vec{N}_{+}\left(\frac{\partial \mu}{\partial c}\right) \vec{\nabla} c+\vec{j} \vec{E}
$$

Using the constitutive relations (19) - (21), Eq. (29) can be transformed into

$c_{p} \rho \partial_{t} T=\vec{\nabla}(\lambda \vec{\nabla} T)+\frac{\vec{j}^{2}}{\kappa}-T \vec{\nabla}(\beta \vec{j})+\left(\frac{\partial \mu}{\partial c}\right) \frac{\left(\vec{N}_{+}-\frac{t_{+}}{F z_{+}} \vec{j}\right)^{2}}{D}-T \vec{\nabla}\left(c\left(\frac{\partial \mu}{\partial c}\right) \frac{k_{T}}{T}\left(\vec{N}_{+}-\frac{t_{+}}{z_{+} F} \vec{j}\right)\right)$

The temperature changes due to thermal conduction and four different sources for heat. They are in the order of their appearance in equation (30): Joule's heat, Thompson effect, heat of mixing and the Soret effect. The Thompson effect can also be written as

$$
T \vec{\nabla}(\beta \vec{j})=T \frac{\partial \beta}{\partial T} \vec{j} \vec{\nabla} T:=\mu_{T} \vec{j} \vec{\nabla} T
$$

Here we used charge conservation (Eq (10)) and the Thompson relation for the Thompson coefficient $\mu_{T}=T \frac{\partial \beta}{\partial T}$. If we neglect all contributions proportional to $k_{T}$ the heat equation (30) reduces further to

$$
c_{p} \rho \partial_{t} T=\vec{\nabla}(\lambda \vec{\nabla} T)+\frac{\vec{j}^{2}}{\kappa}-T \vec{\nabla}(\beta \vec{j})+\left(\frac{\partial \mu}{\partial c}\right) D(\vec{\nabla} c)^{2}
$$

The equations for ion concentration, electric field respective electric potential defined by $\vec{E}=-\vec{\nabla} \Phi$ and temperature can be applied in the electrolyte as well as in the active particles. The only difference is the electric conductivity, which is replaced by the average electronic conductivity $\sigma$ of the solid active particle and carbon black, which is usually used to enhance the electronic conductivity of the electrodes.

\section{$2.2 \quad$ Interface conditions}

To couple the transport in electrolyte and active particles we have to formulate interface conditions, which describe the intercalation and deintercalation of ions. 


\subsubsection{Interface conditions for ionic flux and electric current}

The interface conditions describe the intercalation reaction and the deintercalation reaction respectively on the mesoscopic scale (i.e beyond the scale of the diffuse layer [4]). For one step reactions it is usually assumed that the transport of ions across the interface is completely described by the Butler Volmer approach. More complicated reactions may require the use of more elaborate theories [33], which can be easily incorporated within our theory. Here we use for simplicity the Butler Volmer theory. The current density across the interface $i_{s e}$ due to the intercalation reaction is within the Butler Volmer approach given by $[4]$

$$
i_{s e}=i_{0}\left(\exp \left[\frac{\alpha_{a} F}{R T} \eta_{s}\right]-\exp \left[\frac{-\alpha_{c} F}{R T} \eta_{s}\right]\right)
$$

$\alpha_{A}$ and $\alpha_{C}$ with $\alpha_{A}+\alpha_{C}=1$ are weighting the anodic and the cathodic contribution of the overpotential $\eta_{s}$ to the overall reaction. The overpotential is the deviation of the electrochemical potential from the chemical equilibrium between active particle and electrolyte. It is therefore defined by

$$
\eta_{s}:=\Phi_{s}-\Phi_{e}-\frac{\mu_{e}-\mu_{s}}{z_{+} F}
$$

The overpotential vanishes obviously if the active particle is in equilibrium with the electrolyte. Usually the overpotential is expressed with the help of the half cell open circuit potential $U_{0}$ of the respective electrode relative to a Li metal electrode. Without loss of generality setting $\Phi_{L i}=0$, the open circuit potential $U_{0}$ can be written as

$$
U_{0}=\mu_{L i}-\mu_{s}
$$

Using this expression in Eq. (34) we get

$$
\eta_{s}:=\Phi_{s}-\Phi_{e}-U_{0}-\frac{\mu_{e}-\mu_{L i}}{z_{+} F}
$$

Since the chemical potential of the electrolyte $\mu_{e}$ is very different from the chemical potential $\mu_{L i}$ of the Li metal electrode the last two terms in Eq. (36) do not cancel in general. Introducing the electrochemical potential of the electrolyte $\varphi_{e}$ relative to the chemical potential of $\mathrm{Li}$ - metal

$$
\varphi_{e}:=\Phi_{e}+\frac{\mu_{e}-\mu_{L i}}{z_{+} F}
$$

we can rewrite (36) as

$$
\eta_{s}:=\Phi_{s}-\varphi_{e}-U_{0}
$$


and obtain the standard form of the overpotential but with the electrochemical potential instead of the electric potential.

The amplitude $i_{0}$ in Eq. (33) is given by

$$
i_{0}=k c^{\alpha_{a}} c_{s}^{\alpha_{a}}\left(c_{s, \max }-c_{s}\right)^{\alpha_{c}}
$$

$k$ is a reaction rate. $c_{s, \max }$ is the maximum concentration of ions in the active particle. We assume that $\mathrm{Li}$ ions are not stored in the double layer (i.e. all Li ions are intercalated in the active particle or released into the electrolyte). There should also be no flux of negative charges across the double layer. Neither enter electrons the electrolyte nor intercalate negative ions from the electrolyte in the active particles under ideal conditions. Both effects would lead to degradation [1], which could of course be modeled by modifying the interface conditions. The absence of flux of negative charges across the interface especially means that the total current across the electrolyte-particle interface is due to transport of positive ions only. If the particle is completely filled i.e. $c=c_{s, \max }$, it has to be made

sure by the interface conditions that no electrical current $\vec{j}$ is carried by negative charge carriers across the interface. These conditions can be formulated mathematically in the following way with the normal $\vec{n}$ pointing from the solid into the electrolyte

$$
\begin{aligned}
\overrightarrow{j_{s}} \vec{n} & =i_{s e} \\
\vec{j}_{e} \vec{n} & =i_{s e} \\
\vec{N}_{+, s} \vec{n} & =\frac{i_{s e}}{z_{+} F} \\
\vec{N}_{+, e} \vec{n} & =\frac{i_{s e}}{z_{+} F}
\end{aligned}
$$

\subsubsection{Thermal interface conditions}

To derive the thermal interface conditions we consider a infinitesimally extendend small piece of the interface perpendicular to the normal $\vec{n}$ which points from the active particle into the electrolyte. The interface conditions are most conveniently derived using the form (27) of the heat transport equation. We integrate the temperature balance equation over the infinitesimal small volume element, which contains the whole thickness of double layer. The term proportional to $\vec{\nabla} N_{+}$does not contribute to the flux since the flux is conserved across the double layer (see Eq. (42)). We obtain

$$
\iiint d V c_{p} \rho \partial_{t} T=\iint d A\left(\vec{n}\left(\vec{q}_{s}-\vec{q}_{e}\right)+\vec{n} \vec{N}_{+}\left(\mu_{s}-\mu_{e}\right)+\vec{n} \vec{j}\left(\Phi_{s}-\Phi_{e}\right)\right)
$$


Using Eqs. (40) and (42) and the definition of $\vec{Q}$ we obtain

$$
\iiint d V c_{p} \rho \partial_{t} T=\iint d A\left(\vec{n}\left(\vec{Q}_{s}-\vec{Q}_{e}\right)+\frac{i_{s e}}{z_{+} F}\left(\mu_{s}-\mu_{e}+z_{+} F\left(\Phi_{s}-\Phi_{e}\right)\right)-\frac{i_{s e}}{z_{+} F} T\left(\frac{\partial \mu_{s}}{\partial T}-\frac{\partial \mu_{e}}{\partial T}\right)\right.
$$

If we assume that the double layer instantaneously changes temperature upon changes of the currents, concentrations and temperatures, the double layers is always in a stationary state i.e. the left hand side of Eq. (45) vanishes. Using Eqs. (35) and (37) we obtain

$$
\vec{n}\left(\vec{Q}_{s}-\vec{Q}_{e}\right)=-i_{s e} \eta_{s}-i_{s e} T \frac{\partial U_{0}}{\partial T}-\frac{i_{s e}}{z_{+} F} T \frac{\partial\left(\mu_{e}-\mu_{L i}\right)}{\partial T}
$$

Neglecting the temperature dependence of the chemical potential of Li metal and using the the constitutive relation for Q Eq. (21) we finally obtain

$$
\begin{aligned}
& -\lambda_{s} \vec{n} \vec{\nabla} T_{s}+\lambda_{e} \vec{n} \vec{\nabla} T_{e}= \\
& -i_{s e} \eta_{s}-T i_{s e}\left\{\left(\beta_{s}-\beta_{e}\right)+\frac{\partial\left(U_{0}+\frac{\mu_{e}}{z_{+} F}\right)}{\partial T}\right\}+i_{s e}\left(c_{s} \frac{\partial U_{0}}{\partial T} k_{T, s}+c_{e} \frac{\partial \mu_{e}}{\partial T} \frac{k_{T, e}\left(1-t_{+}\right)}{z_{+} F}(48)\right.
\end{aligned}
$$

Here we also used that the transference number of Li ions in the active particle may be neglected due to the high mobility of electrons compared to the mobility of Li ions. The physical meaning of the various expressions on the right hand side of Eq. (47) is obvious. The first one is the irreversible heat production due to Joule heating. The next term contributes to the reversible Peltier effect and the last one is the Soret effect in the double layer. The Peltier coefficient is given by

$$
\Pi=T\left(\beta_{s}-\beta_{e}\right)+T \frac{\partial\left(U_{0}+\frac{\mu_{e}}{z_{+} F}\right)}{\partial T}
$$

Note that in [11] only the partial derivative of the open circuit potential was given as Peltier coefficient. Our rigorous approach shows naturally that also the differences in the Seebeck coefficients of the two phases and the thermal derivative of the electrolytes chemical potential are contributing to the Peltier coefficient. Since our approach is based on experimentally accessible transport coefficients it is either possible to measure directly the Peltier coefficient [34] or to deduce it from measurements of the open circuit potential, the Seebeck coefficients of the two phases [35] and the thermodynamic derivatives of the chemical potential of the electrolyte. 


\subsection{Closed set of equations for transport in batteries}

\subsubsection{Electrolyte}

The natural variables for the electrolyte are the ion concentration $c$, the electrical potential $\Phi_{e}$ and the temperature $T$. Due to the experimentally motivated formulation of the interface conditions with the open circuit potential $U_{0}$ relative to a $\mathrm{Li}$ metal electrode instead of the difference between chemical potentials of solid particle and electrolyte the electrochemical potential $\varphi$ (see Eq. (37)) had to be introduced. It is therefore more convenient to formulate the transport equations with $\varphi_{e}$ insteadt of $\Phi_{e}$. One has to keep in mind that the electric field is still given by $\vec{E}=-\vec{\nabla} \Phi_{e}=-\vec{\nabla} \varphi_{e}+\vec{\nabla} \mu_{e} /\left(z_{+} F\right)$. The transport equations are than given by

$$
\begin{aligned}
\partial_{t} c_{e}= & \vec{\nabla}\left(D_{e} \vec{\nabla} c_{e}\right)-\vec{\nabla}\left(\frac{t_{+}}{z_{+} F} \vec{j}\right)+\vec{\nabla}\left(\frac{D c_{e} k_{T}}{T} \vec{\nabla} T\right) \\
0= & \vec{\nabla}\left(\kappa \vec{\nabla} \varphi_{e}\right)-\vec{\nabla}\left(\kappa \frac{1-t_{+}}{z_{+} F}\left(\frac{\partial \mu}{\partial c}\right) \vec{\nabla} c_{e}\right)+\vec{\nabla}\left(\kappa\left(\beta_{e}-\frac{1}{z_{+} F}\left(\frac{\partial \mu}{\partial T}\right)\right) \vec{\nabla} T\right) \\
c_{p, e} \rho \partial_{t} T= & \vec{\nabla}\left(\lambda_{e} \vec{\nabla} T\right)+\frac{\vec{j}^{2}}{\kappa}-T \vec{\nabla}\left(\beta_{e} \vec{j}\right)+ \\
& +\left(\frac{\partial \mu}{\partial c}\right) \frac{\left(\vec{N}_{+}-\frac{t_{+}}{F z_{+}} \vec{j}\right)^{2}}{D_{e}}-T \vec{\nabla}\left(c_{e}\left(\frac{\partial \mu}{\partial c}\right) \frac{k_{T, e}}{T}\left(\vec{N}_{+}-\frac{t_{+}}{z_{+} F} \vec{j}\right)\right)
\end{aligned}
$$

Note that the first two equations without the terms proportional to the temperature gradients have the form of the equations for the concentrated electrolyte by Newman [4], but it is important to realize that there exists an important quantitative difference. In the concentrated electrolyte theory of [4] the potential in Eq. (51) is interpreted as the electrical potential $\Phi_{e}$. In our theory the electrical potential had to be replaced by the electrochemical potential $\varphi_{e}$ of the electrolyte, if we use the open circuit potential relative to Li metal in the simple expression for the overpotential Eq. (38).

\subsubsection{Active particles}

Our derivation of the constitutive relations for concentration, electrical potential and temperature is based on general thermodynamic principles and can therefore be applied equally to the transport in active particles. Only if the transport is anisotropical the transport coefficients have to be replaced by tensorial quantities. Here we restrict ourselves to active particles which behave approximatively isotropic on the micrometer scale. The equations are slightly simplified due to the reasonable assumption that the transference 
number can be set to zero, since the electrical current is predominantly electronic of nature. We will denote the electronic conductivity with $\sigma$. The transport equations are then given by

$$
\begin{aligned}
\partial_{t} c_{s} & =\vec{\nabla}\left(D_{s} \vec{\nabla} c_{s}\right)+\vec{\nabla}\left(\frac{D c_{s} k_{T, s}}{T} \vec{\nabla} T\right) \\
0 & =\vec{\nabla}\left(\sigma \vec{\nabla} \Phi_{s}\right)+\vec{\nabla}\left(\beta_{s} \sigma \vec{\nabla} T\right) \\
c_{p, s} \rho \partial_{t} T & =\vec{\nabla}\left(\lambda_{s} \vec{\nabla} T\right)+\frac{\vec{j}^{2}}{\sigma}-T \vec{\nabla}\left(\beta_{s} \vec{j}\right)-z_{+} F \frac{\partial U_{0}}{\partial c} \frac{\vec{N}_{+}^{2}}{D_{s}}+T z_{+} F \vec{\nabla}\left(c_{s} \frac{\partial U_{0}}{\partial c} \frac{k_{T, s}}{T} \vec{N}_{+}\right)(55)
\end{aligned}
$$

Together with the interface conditions (40) - (43) and (47) the complete set of equations for transport in $\mathrm{Li}$ - ion batteries is formulated. Of course the equations have to be closed by using appropriate boundary conditions, but this is standard procedure. They may change from application to application depending on how the cell is coupled to the outside world. Especially different cooling procedure will require different boundary conditions either for the thermal flux or the temperature itself. Also the coupling of the current collector to the electrodes will influence the choice of boundary conditions.

\section{Conclusions}

We have derived the complete set of transport equations on a spatial scale larger than the diffuse double layer in Li ion batteries. Our derivation is based on general principles of nonequilibrium thermodynamics, which guarantee thermodynamic consistency and especially strictly positive entropy production. The equations for transport in the electrolyte differ from the concentrated electrolyte theory of [4]. It can easily be shown [28], that the concentrated electrolyte theory of [4] violates the Onsager relations. But we have also shown above, that the coupled equations for charge and species transport in Li ion batteries (neglecting heat transport) can be transformed in the form used in [3], [4], if in the electrolyte the pure electrical potential is replaced by the electrochemical potential in combination with the definition (38) for the overpotential.

The complete set of equations can either be used to simulate the transport within cells using a spatial representation of the electrodes, which resolves the microstructure of the porous electrodes or as a starting point to obtain a porous media representation of the electrodes as in [15]. In the microscopic approach active particles and electrolyte are treated as separate media. So far this ansatz is only used for simulating the ion and charge transport under isothermal conditions [36, 37]. Inclusion of heat transport is planned as future work. The computational complexity of the problem allows only to 
simulate representative volume elements (REV) of battery cells. But as post processing effective properties of the porous electrode may be obtained as e.g. effective diffusion coefficients or conductivities by numerically averaging over the REV. These quantities may then be used for simulating the whole cell modeled with the porous electrode model.

So far side reactions as e.g. formation of the solid electrolyte interface (SEI) or transport within a SEI have not been considered. Within our approach it is straight forward to include those phenomena. Surface reactions will lead to a modification of the interface conditions. Transport within a SEI requires the addition of another thermodynamic phase with appropriate transport coefficients [38]. Volumetric reactions require the inclusion of the reacting species and the information on the reaction kinetics [20]. The derivation of the transport equations will proceed along the same line as shown above with the only difference that the concentrations of the species are not conserved separately but just the sum of them. In future work we will make extensive use of the model to obtain a better understanding of the coupled transport phenomena and their repercussions on the performance and lifetime of $\mathrm{Li}$ ion batteries.

Acknowledgment. The work was supported by the Fraunhofer system research for electromobility (FSEM) within the economic stimulus package II of the German Ministry of Education and Research. 


\section{References}

[1] J. Vetter, P. Novak, M. R. Wagner, C. Veit, K.-C. Möller, J. O. Besenhard, M. Winter, M. Wohlfahrt-Mehrens, C. Vogler, and A. Hammouche. Ageing mechanisms in lithium-ion batteries. J. Pow. Sources, 147:269-281, 2005.

[2] M. Broussely, Ph. Biensan, F. Bonhomme, Ph. Blanchard, S. Herreyre, K. Nechev, and R. J. Staniewicz. Main aging mechanisms in li ion batteries. J. Pow. Sources, 146:90-96, 2005.

[3] T. F. Fuller, M. Doyle, and J. Newman. Simulation and optimization of the dual lithium ion insertion cell. J. Electrochem. Soc., 141:1-10, 1994.

[4] J. Newman and K. E. Thomas-Alyea. Electrochemical Systems. Wiley, 2004.

[5] K. E. Thomas, J. Newman, and R. M. Darling. Mathematical modeling of lithium batteries. In W. A. Schalkwijk and B. Scrosati, editors, Advances in Lithium-Ion Batteries, pages 345-392. Kluver, 2002.

[6] G. G Botte, V. R. Subramanian, and Ralph E. White. Mathematical modeling of secondary lithium batteries. Electrochimica Acta, 45:2595-2609, 2000.

[7] D. Danilov and P. H. L. Notten. Mathematical modelling of ionic transport in the electrolyte of Li-ion batteries. Electrochimica Acta, 53:5569-5578, 2008.

[8] Laurits H. Olesen, Martin Z. Bazant, and Henrik Bruus. Strongly nonlinear dynamics of electrolytes in large ac voltages. arXiv:0908.3501, 2009.

[9] H. F. Gibbard. Thermal properties of battery systems. J. Electrochem. Soc., 125:353$358,1978$.

[10] D. Bernardi, E. Pawlikowski, and J. Newman. A general energy balance for battery systems. J. Electrochem. Soc., 132:5-12, 1985.

[11] J. Newman. Thermoelectric effects in electrochemical systems. Ind. Eng. Chem. Res., 34:3208-3216, 1995.

[12] C. R. Pals and J. Newman. Thermal modeling of the lithium/polymer battery i.discharge behavior of a single cell. J. Electrochem. Soc., 142:3274-3281, 1995.

[13] L. Rao and J. Newman. Heat generation rate and general energy balance for insertion battery systems. J. Electrochem. Soc., 144:2697-2704, 1997. 
[14] K. E. Thomas-Alyea and J. Newman. Thermal modeling of porous insertion electrodes. J. Electrochem. Soc., 150:A176-A192, 2003.

[15] W. B. Gu and C. Y. Wang. Thermal-electrochemical modeling of battery systems. J. Electrochem. Soc., 147:2910-2922, 2000.

[16] C. Y. Wang, W. B. Gu, and B. Y. Liaw. Micro-macroscopic coupled modeling of batteries and fuel cells. I. model development. J. Electrochem. Soc., 145:3407-3417, 1998.

[17] M. K. Verbrugge. Three-dimensional temperature and current distribution in a battery module. AIChE J., 41:1550-1562, 1995.

[18] H. Huang and T. V. Nguyen. A transient nonisothermal model for valve-regulated Lead-Acid batteries under float. J. Electrochem. Soc., 144:2420-2426, 1997.

[19] P. de Vidts, J. Delgado, B. Wu, D. See, K. Kosanovich, and R. E. White. A nonisothermal Nickel-Hydrogen cell model. J. Electrochem. Soc., 145:3874-3883, 1998.

[20] S.R. de Groot and P. Mazur. Non-Equilibrium Thermodynamics. Dover, 1984.

[21] L. D. Landau and E. M. Lifshitz. Fluid Mechanics. Pergamon, 1959.

[22] L. D. Landau and E. M. Lifshitz. Electrodynamics of Continous Media. Pergamon, Oxford, 1984.

[23] K. Henjes and M. Liu. Hydrodynamics of polarizable liquids. Ann. Physics, 223:243276, 1993.

[24] M. Liu. Hydrodynamic theory of electromagnetic fields in continous media. Phys.Rev.Lett., 70:3580-3583, 1993.

[25] Y. Jiang and M. Liu. Dynamics of dispersive and nonlinear media. Phys.Rev.Lett., 77:1043-1046, 1996.

[26] Y. Jiang and M. Liu. Electromagnetic force in dispersive and transparent media. Phys. Rev. E, 58:6685-6694, 1998.

[27] M. Liu. Fluid dynamics of colloidal and electric liquids. Phys.Rev.Lett., 74:45354538, 1995. 
[28] A. Latz and J. Zausch. Modeling of species and charge transport in Li-ion batteries based on non-equilibrium thermodynamics. Berichte des Fraunhofer ITWM, 190:1-8, 2010 .

[29] M. Z. Bazant, K. Thornton, and A. Ajdari. Diffuse-charge dynamics in electrochemical systems. Phys. Rev. E, 70:021506, 2004.

[30] M. Z. Bazant and K. T. Chu. Nonlinear electric relaxation around conductors. Phys. Rev. E, 74:011501, 2006.

[31] H. J. V. Tyrrell. Thermal Diffusion phenomena in Electrolytes and the Constants Involved, volume 524 of National Bureau of Standards (U.S.) Circular. National Bureau of Standards, 1953.

[32] Jean K. Platten. The soret effect: A review of recent experimental results. Journal of Applied Mechanics, 73(1):5-15, 2006.

[33] A. M. Colclasure and R. J. Kee. Thermodynamically consistent modeling of elementary electrochemistry in Li - ion batteries. Electrochimica Acta, 2010.

[34] Y. V. Kuzminskii and A. V. Gorodyskii. Thermal analysis of electrochemical reactions part i. kinetic method of determining Peltier heats. J. Electroanal. Chem, 252:21-38, 1988.

[35] C. Wood, A. Chmielewski, and D. Zoltan. Measurement of Seebeck coefficient using a large thermal gradient. Rev. Sci. Instrum., 59:951-954, 1988.

[36] C. W. Wang and A. M. Sastry. Mesoscale modeling of Li-ion polymer cell. J. Electrochem. Soc., 154:A1035-A1047, 2007.

[37] J. Zausch, A. Latz, S. Schmidt, G. B. Less, J. H. Seo, S. Han, and A. M. Sastry. Micro-scale modeling of Li-Ion batteries; parameterization and validation. to be published, 2010.

[38] J. Christensen and J. Newman. A mathematical model for Lithium-Ion negative electrode solid electrolyte interphase. J. Electrochem. Soc., 151:A1977-A1988, 2004. 


\section{Published reports of the Fraunhofer ITWM}

The PDF-files of the following reports are available under:

\section{www.itwm.fraunhofer.de/de/ zentral_berichte/berichte}

1. D. Hietel, K. Steiner, J. Struckmeier A Finite - Volume Particle Method for Compressible Flows (19 pages, 1998)

2. M. Feldmann, S. Seibold

Damage Diagnosis of Rotors: Application of Hilbert Transform and Multi-Hypothesis Testing

Keywords: Hilbert transform, damage diagnosis,

Kalman filtering, non-linear dynamics

(23 pages, 1998)

3. Y. Ben-Haim, S. Seibold

Robust Reliability of Diagnostic MultiHypothesis Algorithms: Application to Rotating Machinery

Keywords: Robust reliability, convex models, Kalman filtering, multi-hypothesis diagnosis, rotating machinery, crack diagnosis

(24 pages, 1998)

\section{F.-Th. Lentes, N. Siedow}

Three-dimensional Radiative Heat Transfer in Glass Cooling Processes

(23 pages, 1998)

5. A. Klar, R. Wegener

A hierarchy of models for multilane vehicular traffic

Part I: Modeling

(23 pages, 1998)

Part II: Numerical and stochastic investigations (17 pages, 1998)

\section{A. Klar, N. Siedow}

Boundary Layers and Domain Decomposition for Radiative Heat Transfer and Diffusion Equations: Applications to Glass Manufacturing Processes

(24 pages, 1998)

7. I. Choquet

Heterogeneous catalysis modelling and numerical simulation in rarified gas flows Part l: Coverage locally at equilibrium (24 pages, 1998)

8. J. Ohser, B. Steinbach, C. Lang Efficient Texture Analysis of Binary Images (17 pages, 1998)

9. J. Orlik

Homogenization for viscoelasticity of the integral type with aging and shrinkage (20 pages, 1998)

10. J. Mohring

Helmholtz Resonators with Large Aperture (21 pages, 1998)
11. H. W. Hamacher, A. Schöbel On Center Cycles in Grid Graphs (15 pages, 1998)

12. H. W. Hamacher, K.-H. Küfer Inverse radiation therapy planning a multiple objective optimisation approach (14 pages, 1999)

13. C. Lang, J. Ohser, R. Hilfer On the Analysis of Spatial Binary Images (20 pages, 1999)

14. M. Junk

On the Construction of Discrete Equilibrium Distributions for Kinetic Schemes (24 pages, 1999)

15. M. Junk, S. V. Raghurame Rao

A new discrete velocity method for NavierStokes equations

(20 pages, 1999)

16. H. Neunzert

Mathematics as a Key to Key Technologies (39 pages, 1999)

17. J. Ohser, K. Sandau

Considerations about the Estimation of the Size Distribution in Wicksell's Corpuscle Problem

(18 pages, 1999)

18. E. Carrizosa, H. W. Hamacher, R. Klein, S. Nickel

Solving nonconvex planar location problems by finite dominating sets

Keywords: Continuous Location, Polyhedral Gauges, Finite Dominating Sets, Approximation, Sandwich Algorithm, Greedy Algorithm

(19 pages, 2000)

\section{A. Becker}

A Review on Image Distortion Measures Keywords: Distortion measure, human visual system (26 pages, 2000)

20. H. W. Hamacher, M. Labbé, S. Nickel, T. Sonneborn

Polyhedral Properties of the Uncapacitated Multiple Allocation Hub Location Problem Keywords: integer programming, hub location, facility location, valid inequalities, facets, branch and cut (21 pages, 2000)

21. H. W. Hamacher, A. Schöbel

Design of Zone Tariff Systems in Public Transportation

(30 pages, 2001)

22. D. Hietel, M. Junk, R. Keck, D. Teleaga The Finite-Volume-Particle Method for Conservation Laws (16 pages, 2001)

23. T. Bender, H. Hennes, J. Kalcsics, M. T. Melo, S. Nickel

Location Software and Interface with GIS and Supply Chain Management Keywords: facility location, software development, geographical information systems, supply chain management

(48 pages, 2001)
24. H. W. Hamacher, S. A. Tjandra Mathematical Modelling of Evacuation Problems: A State of Art (44 pages, 2001)

25. J. Kuhnert, S. Tiwari

Grid free method for solving the Poisson equation

Keywords: Poisson equation, Least squares method, Grid free method

(19 pages, 2001)

26. T. Götz, H. Rave, D. Reinel-Bitzer, K. Steiner, $\mathrm{H}$. Tiemeier

Simulation of the fiber spinning process Keywords: Melt spinning, fiber model, Lattice Boltzmann, CFD

(19 pages, 2001)

\section{A. Zemitis}

On interaction of a liquid film with an obstacle Keywords: impinging jets, liquid film, models, numerical solution, shape

22 pages, 2001)

\section{I. Ginzburg, K. Steiner}

Free surface lattice-Boltzmann method to model the filling of expanding cavities by Bingham Fluids

Keywords: Generalized LBE, free-surface phenomena interface boundary conditions, filling processes, Bing ham viscoplastic model, regularized models

(22 pages, 2001)

\section{H. Neunzert}

"Denn nichts ist für den Menschen als Menschen etwas wert, was er nicht mit Leidenschaft tun kann

Vortrag anlässlich der Verleihung des Akademiepreises des Landes RheinlandPfalz am 21.11.2001

Keywords: Lehre, Forschung, angewandte Mathematik, Mehrskalenanalyse, Strömungsmechanik (18 pages, 2001)

30. J. Kuhnert, S. Tiwari

Finite pointset method based on the projection method for simulations of the incompressible Navier-Stokes equations

Keywords: Incompressible Navier-Stokes equations, Meshfree method, Projection method, Particle scheme, Least squares approximation

AMS subject classification: 76D05, 76M28

(25 pages, 2001)

31. R. Korn, M. Krekel

Optimal Portfolios with Fixed Consumption or Income Streams

Keywords: Portfolio optimisation, stochastic control, HJB equation, discretisation of control problems (23 pages, 2002)

32. M. Krekel

Optimal portfolios with a loan dependent credit spread

Keywords: Portfolio optimisation, stochastic control, HJB equation, credit spread, log utility, power utility, non-linear wealth dynamics

(25 pages, 2002)

33. J. Ohser, W. Nagel, K. Schladitz

The Euler number of discretized sets - on the choice of adjacency in homogeneous lattices Keywords: image analysis, Euler number, neighborhod relationships, cuboidal lattice

(32 pages, 2002) 
34. I. Ginzburg, K. Steiner

Lattice Boltzmann Model for Free-Surface flow and Its Application to Filling Process in Casting

Keywords: Lattice Boltzmann models; free-surface phenomena; interface boundary conditions; filling processes; injection molding; volume of fluid method; interface boundary conditions; advection-schemes; upwind-schemes

(54 pages, 2002)

35. M. Günther, A. Klar, T. Materne, R. Wegener

Multivalued fundamental diagrams and stop and go waves for continuum traffic equations Keywords: traffic flow, macroscopic equations, kinetic derivation, multivalued fundamental diagram, stop and go waves, phase transitions

(25 pages, 2002)

36. S. Feldmann, P. Lang, D. Prätzel-Wolters Parameter influence on the zeros of network determinants

Keywords: Networks, Equicofactor matrix polynomials, Realization theory, Matrix perturbation theory

(30 pages, 2002)

\section{K. Koch, J. Ohser, K. Schladitz} Spectral theory for random closed sets and estimating the covariance via frequency space

Keywords: Random set, Bartlett spectrum, fast Fourier transform, power spectrum

(28 pages, 2002)

\section{D. d'Humières, I. Ginzburg}

Multi-reflection boundary conditions for lattice Boltzmann models

Keywords: lattice Boltzmann equation, boudary condistions, bounce-back rule, Navier-Stokes equation

(72 pages, 2002)

\section{R. Korn}

\section{Elementare Finanzmathematik}

Keywords: Finanzmathematik, Aktien, Optionen, Portfolio-Optimierung, Börse, Lehrerweiterbildung, Mathematikunterricht

(98 pages, 2002)

40. J. Kallrath, M. C. Müller, S. Nickel

Batch Presorting Problems:

Models and Complexity Results

Keywords: Complexity theory, Integer programming,

Assigment, Logistics

(19 pages, 2002)

\section{J. Linn}

On the frame-invariant description of the phase space of the Folgar-Tucker equation Key words: fiber orientation, Folgar-Tucker equation, injection molding

(5 pages, 2003)

\section{T. Hanne, S. Nickel}

A Multi-Objective Evolutionary Algorithm for Scheduling and Inspection Planning in Software Development Projects

Key words: multiple objective programming, project management and scheduling, software development, evolutionary algorithms, efficient set

(29 pages, 2003)

43. T. Bortfeld, K.-H. Küfer, M. Monz,

A. Scherrer, C. Thieke, H. Trinkaus

Intensity-Modulated Radiotherapy - A Large Scale Multi-Criteria Programming Problem
Keywords: multiple criteria optimization, representative systems of Pareto solutions, adaptive triangulation, clustering and disaggregation techniques, visualization of Pareto solutions, medical physics, external beam radiotherapy planning, intensity modulated radiotherapy (31 pages, 2003)

\section{T. Halfmann, T. Wichmann}

Overview of Symbolic Methods in Industrial Analog Circuit Design

Keywords: $C A D$, automated analog circuit design, sym bolic analysis, computer algebra, behavioral modeling, system simulation, circuit sizing, macro modeling, differential-algebraic equations, index

(17 pages, 2003)

\section{S. E. Mikhailov, J. Orlik}

Asymptotic Homogenisation in Strength and Fatigue Durability Analysis of Composites

Keywords: multiscale structures, asymptotic homogenization, strength, fatigue, singularity, non-local conditions

(14 pages, 2003)

46. P. Domínguez-Marín, P. Hansen, N. Mladenovic, S. Nickel Heuristic Procedures for Solving the Discrete Ordered Median Problem Keywords: genetic algorithms, variable neighborhood search, discrete facility location

(31 pages, 2003)

47. N. Boland, P. Domínguez-Marín, S. Nickel, J. Puerto

Exact Procedures for Solving the Discrete Ordered Median Problem

Keywords: discrete location, Integer programming

(41 pages, 2003)

\section{S. Feldmann, P. Lang}

Padé-like reduction of stable discrete linear systems preserving their stability

Keywords: Discrete linear systems, model reduction,

stability, Hankel matrix, Stein equation

(16 pages, 2003)

49. J. Kallrath, S. Nickel

A Polynomial Case of the Batch Presorting

\section{Problem}

Keywords: batch presorting problem, online optimization, competetive analysis, polynomial algorithms, logistics (17 pages, 2003)

\section{T. Hanne, H. L. Trinkaus}

knowCube for MCDM -

Visual and Interactive Support for

Multicriteria Decision Making

Key words: Multicriteria decision making, knowledge management, decision support systems, visual interfaces, interactive navigation, real-life applications. (26 pages, 2003)

51. O. lliev, V. Laptev

On Numerical Simulation of Flow Through Oil Filters

Keywords: oil filters, coupled flow in plain and porous media, Navier-Stokes, Brinkman, numerical simulation (8 pages, 2003)

52. W. Dörfler, O. Iliev, D. Stoyanov, D. Vassileva On a Multigrid Adaptive Refinement Solver for Saturated Non-Newtonian Flow in Porous Media

Keywords: Nonlinear multigrid, adaptive refinement, non-Newtonian flow in porous media

(17 pages, 2003)
53. S. Kruse

On the Pricing of Forward Starting Options under Stochastic Volatility

Keywords: Option pricing, forward starting options, Heston model, stochastic volatility, cliquet options (11 pages, 2003)

54. O. Iliev, D. Stoyanov

Multigrid - adaptive local refinement solver for incompressible flows

Keywords: Navier-Stokes equations, incompressible flow projection-type splitting, SIMPLE, multigrid methods, adaptive local refinement, lid-driven flow in a cavity (37 pages, 2003)

\section{V. Starikovicius}

The multiphase flow and heat transfer in porous media

Keywords: Two-phase flow in porous media, various formulations, global pressure, multiphase mixture model, numerical simulation

(30 pages, 2003)

\section{P. Lang, A. Sarishvili, A. Wirsen}

Blocked neural networks for knowledge extraction in the software development process Keywords: Blocked Neural Networks, Nonlinear Regression, Knowledge Extraction, Code Inspection (21 pages, 2003)

\section{H. Knaf, P. Lang, S. Zeiser}

Diagnosis aiding in Regulation

Thermography using Fuzzy Logic

Keywords: fuzzy logic, knowledge representation, expert system

(22 pages, 2003)

58. M. T. Melo, S. Nickel, F. Saldanha da Gama Largescale models for dynamic multicommodity capacitated facility location Keywords: supply chain management, strategic planning, dynamic location, modeling (40 pages, 2003)

\section{J. Orlik}

Homogenization for contact problems with periodically rough surfaces

Keywords: asymptotic homogenization, contact problems (28 pages, 2004)

60. A. Scherrer, K.-H. Küfer, M. Monz,

F. Alonso, T. Bortfeld

IMRT planning on adaptive volume structures - a significant advance of computational complexity

Keywords: Intensity-modulated radiation therapy (IMRT), inverse treatment planning, adaptive volume structures, hierarchical clustering, local refinement, adaptive clustering, convex programming, mesh generation, multi-grid methods

(24 pages, 2004)

\section{D. Kehrwald}

Parallel lattice Boltzmann simulation of complex flows

Keywords: Lattice Boltzmann methods, parallel computing, microstructure simulation, virtual material design, pseudo-plastic fluids, liquid composite moulding (12 pages, 2004)

62. O. Iliev, J. Linn, M. Moog, D. Niedziela, V. Starikovicius

On the Performance of Certain Iterative Solvers for Coupled Systems Arising in Discretization of Non-Newtonian Flow Equations 
Keywords: Performance of iterative solvers, Preconditioners, Non-Newtonian flow

(17 pages, 2004)

\section{R. Ciegis, O. Iliev, S. Rief, K. Steiner}

On Modelling and Simulation of Different Regimes for Liquid Polymer Moulding Keywords: Liquid Polymer Moulding, Modelling, Simulation, Infiltration, Front Propagation, non-Newtonian flow in porous media

(43 pages, 2004)

\section{T. Hanne, H. Neu}

Simulating Human Resources in

\section{Software Development Processes}

Keywords: Human resource modeling, software process, productivity, human factors, learning curve

(14 pages, 2004)

\section{O. Iliev, A. Mikelic, P. Popov}

Fluid structure interaction problems in de-

formable porous media: Toward permeability of deformable porous media

Keywords: fluid-structure interaction, deformable porous media, upscaling, linear elasticity, stokes, finite elements

(28 pages, 2004)

66. F. Gaspar, O. Iliev, F. Lisbona, A. Naumovich, P. Vabishchevich

On numerical solution of 1-D poroelasticity equations in a multilayered domain Keywords: poroelasticity, multilayered material, finite volume discretization, MAC type grid

(41 pages, 2004)

67. J. Ohser, K. Schladitz, K. Koch, M. Nöthe Diffraction by image processing and its application in materials science

Keywords: porous microstructure, image analysis, random set, fast Fourier transform, power spectrum, Bartlett spectrum

(13 pages, 2004)

\section{H. Neunzert}

\section{Mathematics as a Technology: Challenges} for the next 10 Years

Keywords: applied mathematics, technology, modelling, simulation, visualization, optimization, glass processing, spinning processes, fiber-fluid interaction, trubulence effects, topological optimization, multicriteria optimiza tion, Uncertainty and Risk, financial mathematics, Malliavin calculus, Monte-Carlo methods, virtual material design, filtration, bio-informatics, system biology

(29 pages, 2004)

69. R. Ewing, O. lliev, R. Lazarov, A. Naumovich On convergence of certain finite difference discretizations for 1D poroelasticity interface problems

Keywords: poroelasticity, multilayered material, finite volume discretizations, MAC type grid, error estimates (26 pages, 2004 )

70. W. Dörfler, O. Iliev, D. Stoyanov, D. Vassileva On Efficient Simulation of Non-Newtonian Flow in Saturated Porous Media with a Multigrid Adaptive Refinement Solver Keywords: Nonlinear multigrid, adaptive renement non-Newtonian in porous media (25 pages, 2004)

\section{J. Kalcsics, S. Nickel, M. Schröde}

Towards a Unified Territory Design Approach - Applications, Algorithms and GIS Integration Keywords: territory desgin, political districting, sales territory alignment, optimization algorithms, Geographical Information Systems

(40 pages, 2005)
72. K. Schladitz, S Peters, D Reinel-Bitzer, A. Wiegmann, J. Ohser

Design of acoustic trim based on geometric modeling and flow simulation for non-woven Keywords: random system of fibers, Poisson line process, flow resistivity, acoustic absorption, Lattice-Boltzmann method, non-woven (21 pages, 2005)

\section{V. Rutka, A. Wiegmann}

Explicit Jump Immersed Interface Method for virtual material design of the effective elastic moduli of composite materials Keywords: virtual material design, explicit jump immersed interface method, effective elastic moduli, composite materials

(22 pages, 2005)

\section{T. Hanne}

Eine Übersicht zum Scheduling von Baustellen Keywords: Projektplanung, Scheduling, Bauplanung, Bauindustrie

(32 pages, 2005)

75. J. Linn

The Folgar-Tucker Model as a Differetial Algebraic System for Fiber Orientation Calculation

Keywords: fiber orientation, Folgar-Tucker model, invariants, algebraic constraints, phase space, trace stability

(15 pages, 2005)

76. M. Speckert, K. Dreßler, H. Mauch, A. Lion, G. J. Wierda

Simulation eines neuartigen Prüfsystems für Achserprobungen durch MKS-Modellierung einschließlich Regelung Keywords: virtual test rig, suspension testing, multibody simulation, modeling hexapod test rig, opti mization of test rig configuration

(20 pages, 2005)

77. K.-H. Küfer, M. Monz, A. Scherrer, P. Süss, F. Alonso, A. S. A. Sultan, Th. Bortfeld,

D. Craft, Chr. Thieke

Multicriteria optimization in intensity modulated radiotherapy planning Keywords: multicriteria optimization, extreme solutions, real-time decision making, adaptive approxima tion schemes, clustering methods, IMRT planning, reverse engineering

(51 pages, 2005)

78. S. Amstutz, H. Andrä

A new algorithm for topology optimization using a level-set method

Keywords: shape optimization, topology optimization topological sensitivity, level-set

(22 pages, 2005)

\section{N. Ettrich}

Generation of surface elevation models for urban drainage simulation

Keywords: Flooding, simulation, urban elevation models, laser scanning

(22 pages, 2005

80. H. Andrä, J. Linn, I. Matei, I. Shklyar, K. Steiner, E. Teichmann

OPTCAST - Entwicklung adäquater Struk turoptimierungsverfahren für Gießereien Technischer Bericht (KURZFASSUNG) Keywords: Topologieoptimierung, Level-Set-Methode Gießprozesssimulation, Gießtechnische Restriktionen, CAE-Kette zur Strukturoptimierung

(77 pages, 2005)
81. N. Marheineke, R. Wegener Fiber Dynamics in Turbulent Flows Part I: General Modeling Framework Keywords: fiber-fluid interaction; Cosserat rod; turbuence modeling: Kolmogorov's energy spectrum; double-velocity correlations; differentiable Gaussian fields (20 pages, 2005)

\section{Part II: Specific Taylor Drag}

Keywords: flexible fibers; $k-\varepsilon$ turbulence model; fiber-turbulence interaction scales; air drag; random Gaussian aerodynamic force; white noise; stochastic differential equations; ARMA process

(18 pages, 2005)

\section{C. H. Lampert, O. Wirjadi}

An Optimal Non-Orthogonal Separation of the Anisotropic Gaussian Convolution Filter Keywords: Anisotropic Gaussian filter, linear filtering, or entation space, $n D$ image processing, separable filters (25 pages, 2005)

83. H. Andrä, D. Stoyanov

Error indicators in the parallel finite element solver for linear elasticity DDFEM Keywords: linear elasticity, finite element method, hierarchical shape functions, domain decom-position, parallel implementation, a posteriori error estimates (21 pages, 2006)

84. M. Schröder, I. Solchenbach Optimization of Transfer Quality in Regional Public Transit

Keywords: public transit, transfer quality, quadratic assignment problem

(16 pages, 2006

85. A. Naumovich, F. J. Gaspar

On a multigrid solver for the three-dimensional Biot poroelasticity system in multilayered domains

Keywords: poroelasticity, interface problem, multigrid, operator-dependent prolongation

(11 pages, 2006)

86. S. Panda, R. Wegener, N. Marheineke Slender Body Theory for the Dynamics of Curved Viscous Fibers

Keywords: curved viscous fibers; fluid dynamics; NavierStokes equations; free boundary value problem; asymptotic expansions; slender body theory

(14 pages, 2006)

87. E. Ivanov, H. Andrä, A. Kudryavtsev Domain Decomposition Approach for Automatic Parallel Generation of Tetrahedral Grids Key words: Grid Generation, Unstructured Grid, Delau nay Triangulation, Parallel Programming, Domain Decomposition, Load Balancing

(18 pages, 2006)

88. S. Tiwari, S. Antonov, D. Hietel, J. Kuhnert, R. Wegener

A Meshfree Method for Simulations of In teractions between Fluids and Flexible Structures

Key words: Meshfree Method, FPM, Fluid Structure Interaction, Sheet of Paper, Dynamical Coupling (16 pages, 2006)

89. R. Ciegis, O. Iliev, V. Starikovicius, K. Steine Numerical Algorithms for Solving Problems of Multiphase Flows in Porous Media Keywords: nonlinear algorithms, finite-volume method software tools, porous media, flows

16 pages, 2006) 
90. D. Niedziela, O. Iliev, A. Latz

On 3D Numerical Simulations of Viscoelastic Fluids

Keywords: non-Newtonian fluids, anisotropic viscosity, integral constitutive equation

(18 pages, 2006)

\section{A. Winterfeld}

Application of general semi-infinite Programming to Lapidary Cutting Problems Keywords: large scale optimization, nonlinear programming, general semi-infinite optimization, design centering, clustering

(26 pages, 2006)

\section{J. Orlik, A. Ostrovska}

Space-Time Finite Element Approximation and Numerical Solution of Hereditary Linear Viscoelasticity Problems

Keywords: hereditary viscoelasticity; kern approximation by interpolation; space-time finite element approximation, stability and a priori estimate

(24 pages, 2006)

93. V. Rutka, A. Wiegmann, H. Andrä EJIIM for Calculation of effective Elastic Moduli in 3D Linear Elasticity

Keywords: Elliptic PDE, linear elasticity, irregular domain, finite differences, fast solvers, effective elastic moduli

(24 pages, 2006)

\section{A. Wiegmann, A. Zemitis}

EJ-HEAT: A Fast Explicit Jump Harmonic Averaging Solver for the Effective Heat Conductivity of Composite Materials Keywords: Stationary heat equation, effective thermal conductivity, explicit jump, discontinuous coefficients, virtual material design, microstructure simulation, EJ-HEAT

(21 pages, 2006)

\section{A. Naumovich}

On a finite volume discretization of the three-dimensional Biot poroelasticity system in multilayered domains

Keywords: Biot poroelasticity system, interface problems, finite volume discretization, finite difference method (21 pages, 2006)

\section{M. Krekel, J. Wenzel}

A unified approach to Credit Default Swaption and Constant Maturity Credit Default Swap valuation

Keywords: LIBOR market model, credit risk, Credit Default Swaption, Constant Maturity Credit Default Swapmethod

(43 pages, 2006)

\section{A. Dreyer}

\section{Interval Methods for Analog Circiuts}

Keywords: interval arithmetic, analog circuits, tolerance analysis, parametric linear systems, frequency response, symbolic analysis, CAD, computer algebra

(36 pages, 2006)

98. N. Weigel, S. Weihe, G. Bitsch, K. Dreßler Usage of Simulation for Design and Optimization of Testing

Keywords: Vehicle test rigs, MBS, control, hydraulics,

testing philosophy

(14 pages, 2006)

99. H. Lang, G. Bitsch, K. Dreßler, M. Speckert Comparison of the solutions of the elastic and elastoplastic boundary value problems
Keywords: Elastic BVP, elastoplastic BVP, variational inequalities, rate-independency, hysteresis, linear kinematic hardening, stop- and play-operator

(21 pages, 2006)

100. M. Speckert, K. Dreßler, H. Mauch MBS Simulation of a hexapod based suspension test rig

Keywords: Test rig, MBS simulation, suspension,

hydraulics, controlling, design optimization

(12 pages, 2006)

101. S. Azizi Sultan, K.-H. Küfer

A dynamic algorithm for beam orientations in multicriteria IMRT planning

Keywords: radiotherapy planning, beam orientation optimization, dynamic approach, evolutionary algorithm, global optimization

(14 pages, 2006)

102. T. Götz, A. Klar, N. Marheineke, R. Wegener A Stochastic Model for the Fiber Lay-down Process in the Nonwoven Production Keywords: fiber dynamics, stochastic Hamiltonian system, stochastic averaging

(17 pages, 2006)

103. Ph. Süss, K.-H. Küfer

Balancing control and simplicity: a variable aggregation method in intensity modulated radiation therapy planning

Keywords: IMRT planning, variable aggregation, clustering methods

(22 pages, 2006)

104. A. Beaudry, G. Laporte, T. Melo, S. Nickel Dynamic transportation of patients in hospitals

Keywords: in-house hospital transportation, dial-a-ride, dynamic mode, tabu search

(37 pages, 2006)

105. Th. Hanne

Applying multiobjective evolutionary algorithms in industrial projects

Keywords: multiobjective evolutionary algorithms, discrete optimization, continuous optimization, electronic circuit design, semi-infinite programming, scheduling (18 pages, 2006)

106. J. Franke, S. Halim

Wild bootstrap tests for comparing signals and images

Keywords: wild bootstrap test, texture classification, textile quality control, defect detection, kernel estimate, nonparametric regression

(13 pages, 2007)

107. Z. Drezner, S. Nickel

Solving the ordered one-median problem in the plane

Keywords: planar location, global optimization, ordered median, big triangle small triangle method, bounds, numerical experiments

(21 pages, 2007)

108. Th. Götz, A. Klar, A. Unterreiter, R. Wegener

Numerical evidance for the non-existing of solutions of the equations desribing rotational fiber spinning

Keywords: rotational fiber spinning, viscous fibers, boundary value problem, existence of solutions (11 pages, 2007)
109. Ph. Süss, K.-H. Küfer

Smooth intensity maps and the BortfeldBoyer sequencer

Keywords: probabilistic analysis, intensity modulated radiotherapy treatment (IMRT), IMRT plan application step-and-shoot sequencing

(8 pages, 2007)

110. E. Ivanov, O. Gluchshenko, H. Andrä, A. Kudryavtsev

Parallel software tool for decomposing and meshing of $3 d$ structures

Keywords: a-priori domain decomposition, unstructured grid, Delaunay mesh generation

(14 pages, 2007)

111. O. lliev, R. Lazarov, J. Willems Numerical study of two-grid preconditioners for 1d elliptic problems with highly oscillating discontinuous coefficients Keywords: two-grid algorithm, oscillating coefficients, preconditioner

(20 pages, 2007)

112. L. Bonilla, T. Götz, A. Klar, N. Marheineke, R. Wegener

Hydrodynamic limit of the Fokker-Planckequation describing fiber lay-down processes

Keywords: stochastic dierential equations, FokkerPlanck equation, asymptotic expansion, Ornstein-

Uhlenbeck process

(17 pages, 2007)

\section{S. Rief}

Modeling and simulation of the pressing section of a paper machine

Keywords: paper machine, computational fluid dynamics, porous media

(41 pages, 2007)

114. R. Ciegis, O. Iliev, Z. Lakdawala

On parallel numerical algorithms for simulating industrial filtration problems

Keywords: Navier-Stokes-Brinkmann equations, finite volume discretization method, SIMPLE, parallel computing, data decomposition method

(24 pages, 2007)

\section{N. Marheineke, R. Wegener}

Dynamics of curved viscous fibers with surface tension

Keywords: Slender body theory, curved viscous bers with surface tension, free boundary value problem (25 pages, 2007)

116. S. Feth, J. Franke, M. Speckert Resampling-Methoden zur mse-Korrektur und Anwendungen in der Betriebsfestigkeit Keywords: Weibull, Bootstrap, Maximum-Likelihood, Betriebsfestigkeit

(16 pages, 2007)

117. H. Knaf

Kernel Fisher discriminant functions - a concise and rigorous introduction

Keywords: wild bootstrap test, texture classification, textile quality control, defect detection, kernel estimate, nonparametric regression

(30 pages, 2007)

118. O. Iliev, I. Rybak

On numerical upscaling for flows in heterogeneous porous media 
Keywords: numerical upscaling heterogeneous porous media, single phase flow, Darcy's law, multiscale problem, effective permeability, multipoint flux approximation, anisotropy

(17 pages, 2007)

\section{O. Iliev, I. Rybak}

On approximation property of multipoint flux approximation method

Keywords: Multipoint flux approximation, finite volume method, elliptic equation, discontinuous tensor coeffi-

cients, anisotropy

(15 pages, 2007)

120. O. Iliev, I. Rybak, J. Willems

On upscaling heat conductivity for a class of industrial problems

Keywords: Multiscale problems, effective heat conductivity, numerical upscaling, domain decomposition

(21 pages, 2007)

121. R. Ewing, O. Iliev, R. Lazarov, I. Rybak On two-level preconditioners for flow in porous media

Keywords: Multiscale problem, Darcy's law, single phase flow, anisotropic heterogeneous porous media, numerical upscaling, multigrid, domain decomposition, efficient preconditioner

(18 pages, 2007)

122. M. Brickenstein, A. Dreyer

POLYBORI: A Gröbner basis framework for Boolean polynomials

Keywords: Gröbner basis, formal verification, Boolean polynomials, algebraic cryptoanalysis, satisfiability

(23 pages, 2007)

\section{O. Wirjad}

Survey of $3 d$ image segmentation methods Keywords: image processing, 3d, image segmentation, binarization

(20 pages, 2007)

\section{S. Zeytun, A. Gupta}

A Comparative Study of the Vasicek and the CIR Model of the Short Rate

Keywords: interest rates, Vasicek model, CIR-model,

calibration, parameter estimation

(17 pages, 2007)

\section{G. Hanselmann, A. Sarishvili}

Heterogeneous redundancy in software quality prediction using a hybrid Bayesian approach

Keywords: reliability prediction, fault prediction, nonhomogeneous poisson process, Bayesian model averaging

(17 pages, 2007)

126. V. Maag, M. Berger, A. Winterfeld, K.-H Küfer

A novel non-linear approach to minimal area rectangular packing

Keywords: rectangular packing, non-overlapping constraints, non-linear optimization, regularization, relaxation

(18 pages, 2007)

127. M. Monz, K.-H. Küfer, T. Bortfeld, C. Thieke Pareto navigation - systematic multi-criteria-based IMRT treatment plan determination

Keywords: convex, interactive multi-objective optimization, intensity modulated radiotherapy planning

(15 pages, 2007)
128. M. Krause A Scherrer

On the role of modeling parameters in IMRT plan optimization

Keywords: intensity-modulated radiotherapy (IMRT) inverse IMRT planning, convex optimization, sensitivity analysis, elasticity, modeling parameters, equivalent uniform dose (EUD)

(18 pages, 2007)

\section{A. Wiegmann}

Computation of the permeability of porous materials from their microstructure by FFFStokes

Keywords: permeability, numerical homogenization

fast Stokes solver

(24 pages, 2007)

130. T. Melo, S. Nickel, F. Saldanha da Gama Facility Location and Supply Chain Management - A comprehensive review

Keywords: facility location, supply chain management network design

(54 pages, 2007)

131. T. Hanne, T. Melo, S. Nickel

Bringing robustness to patient flow management through optimized patient transports in hospitals

Keywords: Dial-a-Ride problem, online problem, case study, tabu search, hospital logistics

(23 pages, 2007)

132. R. Ewing, O. Iliev, R. Lazarov, I. Rybak, J. Willems

An efficient approach for upscaling properties of composite materials with high contrast of coefficients

Keywords: effective heat conductivity, permeability of fractured porous media, numerical upscaling, fibrous insulation materials, metal foams

(16 pages, 2008)

133. S. Gelareh, S. Nickel

New approaches to hub location problems in public transport planning Keywords: integer programming, hub location, transportation, decomposition, heuristic

(25 pages, 2008)

134. G. Thömmes, J. Becker, M. Junk, A. K. Vaikuntam, D. Kehrwald, A. Klar, K. Steiner, A. Wiegmann

A Lattice Boltzmann Method for immiscible multiphase flow simulations using the Level Set Method

Keywords: Lattice Boltzmann method, Level Set method, free surface, multiphase flow (28 pages, 2008

\section{J. Orlik}

Homogenization in elasto-plasticity

Keywords: multiscale structures, asymptotic homogenization, nonlinear energy

(40 pages, 2008)

136. J. Almquist, H. Schmidt, P. Lang, J. Deitmer, M. Jirstrand, D. Prätzel-Wolters, H. Becker

Determination of interaction between MCT1 and CAII via a mathematical and physiological approach

Keywords: mathematical modeling; model reduction: electrophysiology; $\mathrm{pH}$-sensitive microelectrodes; proton antenna

(20 pages, 2008)
137. E. Savenkov, H. Andrä, O. Iliev

An analysis of one regularization approach for solution of pure Neumann problem Keywords: pure Neumann problem, elasticity, regularization, finite element method, condition number (27 pages, 2008)

138. O. Berman, J. Kalcsics, D. Krass, S. Nickel The ordered gradual covering location problem on a network

Keywords: gradual covering, ordered median function, network location

(32 pages, 2008)

139. S. Gelareh, S. Nickel

Multi-period public transport design: A novel model and solution approaches Keywords: Integer programming, hub location, public transport, multi-period planning, heuristics (31 pages, 2008)

140. T. Melo, S. Nickel, F. Saldanha-da-Gama Network design decisions in supply chain planning

Keywords: supply chain design, integer programming models, location models, heuristics

(20 pages, 2008)

141. C. Lautensack, A. Särkkä, J. Freitag K. Schladitz

Anisotropy analysis of pressed point processes

Keywords: estimation of compression, isotropy test nearest neighbour distance, orientation analysis, polar ice, Ripley's K function

(35 pages, 2008)

142. O. Iliev, R. Lazarov, J. Willems

A Graph-Laplacian approach for calculating the effective thermal conductivity of complicated fiber geometries

Keywords: graph laplacian, effective heat conductivity, numerical upscaling, fibrous materials

(14 pages, 2008)

143. J. Linn, T. Stephan, J. Carlsson, R. Bohlin Fast simulation of quasistatic rod deformations for VR applications

Keywords: quasistatic deformations, geometrically exact rod models, variational formulation, energy minimization, finite differences, nonlinear conjugate gradients

(7 pages, 2008)

\section{J. Linn, T. Stephan}

Simulation of quasistatic deformations using discrete rod models

Keywords: quasistatic deformations, geometrically exact rod models, variational formulation, energy minimization, finite differences, nonlinear conjugate gradients

(9 pages, 2008

145. J. Marburger, N. Marheineke, R. Pinnau Adjoint based optimal control using meshless discretizations

Keywords: Mesh-less methods, particle methods, Eulerian-Lagrangian formulation, optimization strategies, adjoint method, hyperbolic equations

(14 pages, 2008

\section{S. Desmettre, J. Gould, A. Szimayer}

Own-company stockholding and work effort preferences of an unconstrained executive Keywords: optimal portfolio choice, executive compensation

(33 pages, 2008) 
147. M. Berger, M. Schröder, K.-H. Küfer A constraint programming approach for the two-dimensional rectangular packing problem with orthogonal orientations

Keywords: rectangular packing, orthogonal orientations non-overlapping constraints, constraint propagation

(13 pages, 2008)

148. K. Schladitz, C. Redenbach, T. Sych, M. Godehardt

Microstructural characterisation of open foams using $3 d$ images

Keywords: virtual material design, image analysis, open foams

(30 pages, 2008)

149. E. Fernández, J. Kalcsics, S. Nickel, R. Ríos-Mercado

A novel territory design model arising in the implementation of the WEEE-Directive Keywords: heuristics, optimization, logistics, recycling (28 pages, 2008)

150. H. Lang, J. Linn

Lagrangian field theory in space-time for geometrically exact Cosserat rods Keywords: Cosserat rods, geometrically exact rods, small strain, large deformation, deformable bodies, Lagrangian field theory, variational calculus (19 pages, 2009)

151. K. Dreßler, M. Speckert, R. Müller, Ch. Weber

Customer loads correlation in truck engineering

Keywords: Customer distribution, safety critical components, quantile estimation, Monte-Carlo methods (11 pages, 2009)

152. H. Lang, K. Dreßler

An improved multiaxial stress-strain correction model for elastic FE postprocessing Keywords: Jiang's model of elastoplasticity, stress-strain correction, parameter identification, automatic differentiation, least-squares optimization, Coleman-Li algorithm

(6 pages, 2009)

153. J. Kalcsics, S. Nickel, M. Schröder A generic geometric approach to territory design and districting

Keywords: Territory design, districting, combinatorial optimization, heuristics, computational geometry (32 pages, 2009)

154. Th. Fütterer, A. Klar, R. Wegener An energy conserving numerical scheme for the dynamics of hyperelastic rods

Keywords: Cosserat rod, hyperealstic, energy conservation, finite differences

(16 pages, 2009)

155. A. Wiegmann, L. Cheng, E. Glatt, O. Iliev, S. Rief

Design of pleated filters by computer simulations

Keywords: Solid-gas separation, solid-liquid separation, pleated filter, design, simulation

(21 pages, 2009)

156. A. Klar, N. Marheineke, R. Wegener Hierarchy of mathematical models for production processes of technical textiles
Keywords: Fiber-fluid interaction, slender-body theory, turbulence modeling, model reduction, stochastic differential equations, Fokker-Planck equation, asymptotic expansions, parameter identification

(21 pages, 2009)

157. E. Glatt, S. Rief, A. Wiegmann, M. Knefel, E. Wegenke

Structure and pressure drop of real and virtual metal wire meshes

Keywords: metal wire mesh, structure simulation, model calibration, CFD simulation, pressure loss (7 pages, 2009)

\section{S. Kruse, M. Mülle}

Pricing American call options under the assumption of stochastic dividends - An application of the Korn-Rogers model Keywords: option pricing, American options, dividends, dividend discount model, Black-Scholes model (22 pages, 2009)

159. H. Lang, J. Linn, M. Arnold

Multibody dynamics simulation of geometrically exact Cosserat rods

Keywords: flexible multibody dynamics, large deformations, finite rotations, constrained mechanical systems, structural dynamics

(20 pages, 2009)

160. P. Jung, S. Leyendecker, J. Linn, M. Ortiz Discrete Lagrangian mechanics and geometrically exact Cosserat rods

Keywords: special Cosserat rods, Lagrangian mechanics, Noether's theorem, discrete mechanics, frame-indifference, holonomic constraints

(14 pages, 2009)

161. M. Burger, K. Dreßler, A. Marquardt, M. Speckert

Calculating invariant loads for system simulation in vehicle engineering

Keywords: iterative learning control, optimal control

theory, differential algebraic equations (DAEs)

(18 pages, 2009)

162. M. Speckert, N. Ruf, K. Dreßler Undesired drift of multibody models excited by measured accelerations or forces Keywords: multibody simulation, full vehicle model, force-based simulation, drift due to noise (19 pages, 2009)

163. A. Streit, K. Dreßler, M. Speckert, J. Lichter, T. Zenner, P. Bach

Anwendung statistischer Methoden zur Erstellung von Nutzungsprofilen für die Auslegung von Mobilbaggern

Keywords: Nutzungsvielfalt, Kundenbeanspruchung, Bemessungsgrundlagen

(13 pages, 2009)

164. I. Correia, S. Nickel, F. Saldanha-da-Gama The capacitated single-allocation hub location problem revisited: A note on a classical formulation

Keywords: Capacitated Hub Location, MIP formulations (10 pages, 2009)

165. F. Yaneva, T. Grebe, A. Scherrer

An alternative view on global radiotherapy optimization problems

Keywords: radiotherapy planning, path-connected sublevelsets, modified gradient projection method, improving and feasible directions

(14 pages, 2009)
166. J. I. Serna, M. Monz, K.-H. Küfer, C. Thieke Trade-off bounds and their effect in multicriteria IMRT planning

Keywords: trade-off bounds, multi-criteria optimization, IMRT, Pareto surface

(15 pages, 2009)

167. W. Arne, N. Marheineke, A. Meister, R. Wegener

Numerical analysis of Cosserat rod and string models for viscous jets in rotational spinning processes

Keywords: Rotational spinning process, curved viscous fibers, asymptotic Cosserat models, boundary value problem, existence of numerical solutions

(18 pages, 2009)

168. T. Melo, S. Nickel, F. Saldanha-da-Gama An LP-rounding heuristic to solve a multiperiod facility relocation problem

Keywords: supply chain design, heuristic, linear programming, rounding

(37 pages, 2009)

169. I. Correia, S. Nickel, F. Saldanha-da-Gama Single-allocation hub location problems with capacity choices

Keywords: hub location, capacity decisions, MILP formulations

(27 pages, 2009)

170. S. Acar, K. Natcheva-Acar

A guide on the implementation of the

Heath-Jarrow-Morton Two-Factor Gaussian

Short Rate Model (HJM-G2++)

Keywords: short rate model, two factor Gaussian,

G2++, option pricing, calibration

(30 pages, 2009)

171. A. Szimayer, G. Dimitroff, S. Lorenz A parsimonious multi-asset Heston model: calibration and derivative pricing

Keywords: Heston model, multi-asset, option pricing, calibration, correlation

(28 pages, 2009)

\section{N. Marheineke, R. Wegener}

Modeling and validation of a stochastic drag for fibers in turbulent flows

Keywords: fiber-fluid interactions, long slender fibers, turbulence modelling, aerodynamic drag, dimensional analysis, data interpolation, stochastic partial differential algebraic equation, numerical simulations, experimental validations

(19 pages, 2009)

173. S. Nickel, M. Schröder, J. Steeg Planning for home health care services Keywords: home health care, route planning, metaheuristics, constraint programming

(23 pages, 2009)

174. G. Dimitroff, A. Szimayer, A. Wagner Quanto option pricing in the parsimonious Heston model

Keywords: Heston model, multi asset, quanto options, option pricing

(14 pages, 2009) 174. G. Dimitroff, A. Szimayer, A. Wagner

175. S. Herkt, K. Dreßler, R. Pinnau

Model reduction of nonlinear problems in structural mechanics

Keywords: flexible bodies, FEM, nonlinear model reduction, POD

(13 pages, 2009) 
176. M. K. Ahmad, S. Didas, J. Iqbal

Using the Sharp Operator for edge detection and nonlinear diffusion

Keywords: maximal function, sharp function, image processing, edge detection, nonlinear diffusion

(17 pages, 2009)

177. M. Speckert, N. Ruf, K. Dreßler, R. Müller, C. Weber, S. Weihe

Ein neuer Ansatz zur Ermittlung von Erprobungslasten für sicherheitsrelevante Bauteile

Keywords: sicherheitsrelevante Bauteile, Kundenbeanspruchung, Festigkeitsverteilung, Ausfallwahrscheinlichkeit, Konfidenz, statistische Unsicherheit, Sicher-

heitsfaktoren

(16 pages, 2009)

178. J. Jegorovs

Wave based method: new applicability areas Keywords: Elliptic boundary value problems, inhomogeneous Helmholtz type differential equations in bounded domains, numerical methods, wave based method, uniform $B$-splines

(10 pages, 2009)

179. H. Lang, M. Arnold

Numerical aspects in the dynamic simulation of geometrically exact rods Keywords: Kirchhoff and Cosserat rods, geometrically exact rods, deformable bodies, multibody dynamics, artial differential algebraic equations, method of lines, time integration

(21 pages, 2009)

180. H. Lang

Comparison of quaternionic and rotationfree null space formalisms for multibody dynamics

Keywords: Parametrisation of rotations, differentialalgebraic equations, multibody dynamics, constrained mechanical systems, Lagrangian mechanics

(40 pages, 2010)

181. S. Nickel, F. Saldanha-da-Gama, H.-P. Ziegler Stochastic programming approaches for risk aware supply chain network design problems Keywords: Supply Chain Management, multi-stage stochastic programming, financial decisions, risk (37 pages, 2010)

182. P. Ruckdeschel, N. Horbenko

Robustness properties of estimators in generalized Pareto Models

Keywords: global robustness, local robustness, finite

sample breakdown point, generalized Pareto distribution

(58 pages, 2010)

183. P. Jung, S. Leyendecker, J. Linn, M. Ortiz

A discrete mechanics approach to Cosserat rod theory - Part 1: static equilibria

Keywords: Special Cosserat rods; Lagrangian mechanics; Noether's theorem; discrete mechanics; frameindifference; holonomic constraints; variational formulation

(35 pages, 2010)

184. R. Eymard, G. Printsypar

A proof of convergence of a finite volume scheme for modified steady Richards' equation describing transport processes in the pressing section of a paper machine Keywords: flow in porous media, steady Richards' equation, finite volume methods, convergence of approximate solution

(14 pages, 2010)

185. P. Ruckdeschel

\section{Optimally Robust Kalman Filtering}

Keywords: robustness, Kalman Filter, innovation outlier, additive outlier

(42 pages, 2010)

186. S. Repke, N. Marheineke, R. Pinnau

On adjoint-based optimization of a free surface Stokes flow

Keywords: film casting process, thin films, free surface

Stokes flow, optimal control, Lagrange formalism

(13 pages, 2010)

187. O. Iliev, R. Lazarov, J. Willems

Variational multiscale Finite Element

Method for flows in highly porous media

Keywords: numerical upscaling, flow in heterogeneous porous media, Brinkman equations, Darcy's law, subgrid approximation, discontinuous Galerkin mixed FEM

(21 pages, 2010)

188. S. Desmettre, A. Szimayer

Work effort, consumption, and portfolio selection: When the occupational choice matters

Keywords: portfolio choice, work effort, consumption, occupational choice

(34 pages, 2010)

189. O. Iliev, Z. Lakdawala, V. Starikovicius

On a numerical subgrid upscaling algorithm for Stokes-Brinkman equations

Keywords: Stokes-Brinkman equations, subgrid

approach, multiscale problems, numerical upscaling

(27 pages, 2010)

190. A. Latz, J. Zausch, O. Iliev

Modeling of species and charge transport in

Li-Ion Batteries based on non-equilibrium thermodynamics

Keywords: lithium-ion battery, battery modeling, elec trochemical simulation, concentrated electrolyte, ion transpor

(8 pages, 2010)

191. P. Popov, Y. Vutov, S. Margenov, O. Iliev Finite volume discretization of equations describing nonlinear diffusion in Li-lon batteries

Keywords: nonlinear diffusion, finite volume discretization, Newton method, Li-lon batteries

(9 pages, 2010)

192. W. Arne, N. Marheineke, R. Wegener Asymptotic transition from Cosserat rod to string models for curved viscous inertial jets

Keywords: rotational spinning processes; inertial and viscous-inertial fiber regimes; asymptotic limits; slenderbody theory; boundary value problems

(23 pages, 2010)

193. L. Engelhardt, M. Burger, G. Bitsch Real-time simulation of multibody-systems for on-board applications

Keywords: multibody system simulation, real-time simulation, on-board simulation, Rosenbrock methods (10 pages, 2010)

194. M. Burger, M. Speckert, K. Dreßler Optimal control methods for the calculation of invariant excitation signals for multibody systems

Keywords: optimal control, optimization, mbs simula-

tion, invariant excitation

(9 pages, 2010)
195. A. Latz, J. Zausch

Thermodynamic consistent transport theory of Li-lon batteries

Keywords: Li-Ion batteries, nonequilibrium thermodynamics, thermal transport, modeling (18 pages, 2010)

Status quo: August 2010 